\title{
Relationship between gastroesophageal reflux disease and laparoscopic sleeve gastrectomy: a narrative review
}

\author{
Flavius Mocian, Marius Coroș \\ Third Department of Surgery, “George Emil Palade” University of Medicine, Pharmacy, Science and Technology, Târgu Mureș, Romania \\ Videosurgery Miniinv 2021; 16 (4): 648-655 \\ DOI: https://doi.org/10.5114/wiitm.2021.103948
}

\begin{abstract}
This paper aims to revisit the relationship between gastroesophageal reflux disease and laparoscopic sleeve gastrectomy in obese patients by reviewing the recent available literature, in the form of a narrative review. The interpretation of the current evidence is challenged by the fact that published data are contradictory and comparison between studies is difficult. Most studies investigate the presence of gastroesophageal reflux disease by assessing only the symptoms reported by patients. A few studies have objectively investigated gastroesophageal reflux disease by functional tests and endoscopic evaluation. Also, the surgical technique of laparoscopic sleeve gastrectomy plays an important role in the incidence of postoperative gastroesophageal reflux disease. In conclusion, surgeons must be aware of the existence of gastroesophageal reflux disease and, at the same time, patients should be informed regarding the possible effect of laparoscopic sleeve gastrectomy on gastroesophageal reflux disease.
\end{abstract}

Key words: gastroesophageal reflux disease, laparoscopic sleeve gastrectomy, obesity, esophageal function tests, esophagogastroduodenoscopy.

\section{Introduction}

Gastroesophageal reflux disease (GERD) represents a disorder of the superior gastrointestinal tract that is defined by heartburn and regurgitation, which develops when reflux of the stomach contents causes troublesome symptoms and/or complications, according to the Evidence-Based Consensus of the Montreal Definition and Classification of Gastroesophageal Reflux Disease. Apart from heartburn and regurgitation, symptoms include dysphagia, chest pain, shortness of breath, cough, and hoarseness [1].

GERD is one of the most prevalent gastrointestinal disorders, with approximately $15-20 \%$ of the western adult population experiencing heartburn and/or regurgitation at least once a week. It is the most common indication for patients to seek a gastroenterology unit $[2,3]$.
Obesity represents one of the most important risk factors for GERD and, with a sharp increase in the number of obese patients globally, the incidence of GERD has significantly increased [4]. Obesity is defined as disproportionate body weight for height with an excessive accumulation of adipose tissue that is accompanied by chronic, systemic inflammation. In numbers, obesity is classified as a body mass index (BMI) greater than $30 \mathrm{~kg} / \mathrm{m}^{2}$, and morbid obesity is classified as a BMI greater than $40 \mathrm{~kg} / \mathrm{m}^{2}$. Obesity has become one of the most threatening global public health problems and can be declared a pandemic of the $21^{\text {st }}$ century $[5,6]$.

Studies have shown that elevated BMI plays a role in the incompetence of the gastroesophageal junction and that weight loss reduces the symptoms of GERD. Bariatric surgery is the solution of choice for 
obese patients who have failed lifestyle and dietary modifications as well as pharmacologic substances. It has been shown to be the most efficient means of achieving sustainable weight loss. Bariatric surgery is currently recommended for obese patients who have a BMI higher than $40 \mathrm{~kg} / \mathrm{m}^{2}$ or more than $35 \mathrm{~kg} / \mathrm{m}^{2}$ with comorbidities. Is considered an effective treatment for obesity and related comorbidities, but there is currently a lack of standard surgical procedures, especially if GERD is present [7]. In theory, bariatric surgery should result in reduced GERD through facilitating sustainable weight loss, but as we are gaining information about the different complications after bariatric surgery, GERD remains an important subject among bariatric surgeons.

The most commonly performed bariatric surgery worldwide is the laparoscopic sleeve gastrectomy (LSG). LSG proved to be efficient in weight loss and has gained popularity because of its advantages. It is a relatively simple procedure, which does not involve anatomical rearrangement or surgical anastomoses and has a shorter operative time. It also appears safer in the long term with a lower risk of marginal ulceration, internal hernias, or adverse nutritional consequences [8, 9]. However, as LSG's popularity continues to increase, one major drawback is the potential development or worsening of GERD postoperatively [10].

\section{Aim}

This paper aims to revisit the relationship between GERD and LSG in obese patients by reviewing the recent available literature concerning this subject, in the form of a narrative [11] review.

\section{Gastroesophageal reflux disease and obesity}

As mentioned in the introduction, obesity is known to play a role in the development of GERD, so this topic generates great interest among bariatric surgeons. A meta-analysis conducted by Hampel et al. described the magnitude of obesity and the risk for GERD development. In nine studies evaluating the impact of obesity on GERD, six of these studies found statistically significant associations between obesity measured by BMI values and the prevalence of GERD [4]. At the same time, obesity is associated with complications related to longstanding GERD such as erosive esophagitis (EE) and Bar- rett's esophagus (BE). BE represents a premalignant condition of the lower esophagus in which the normal squamous epithelial cells undergo metaplasia, becoming columnar epithelial cells, resulting in increasing patients' risk for esophageal adenocarcinoma [12]. BE can be diagnosed in approximately $1.2 \%$ of non-obese patients, while the incidence rises to $9 \%$ in obese patients [13].

The pathophysiology of GERD in an obese population is multifactorial.

The abdominal pressure is increased in obese patients and it correlates directly with BMI. For each unit increase in BMI, there was approximately a $10 \%$ increase in intragastric pressure that is likely to be related to the volume of abdominal fat resulting in mechanical pressure on the stomach and an altered transdiaphragmatic pressure gradient [14].

In obese patients there exists a high prevalence of defective lower esophageal sphincter (LES) [15, 16], probably owing to the increased presence of a concomitant hiatal hernia $(\mathrm{HH})$ [17]. In an observational study of 1659 patients, Ayazi et al. found that increased BMI was associated with both mechanical dysfunctions of the LES and esophageal acid exposure; in particular, $13 \%$ of underweight patients had a defective LES, whereas $50 \%$ of overweight and $55 \%$ of obese patients had defective LES [15].

Transient LES relaxation (TLESR) was found to be more frequent and more closely associated with reflux in obese patients with a dose-effect relation to BMI. Also, TLESR seems to occur more frequently when an $\mathrm{HH}$ is also present [14]. Obese patients also have improper esophageal clearance and dysmotility of the esophagus [16].

All these mechanisms play a major role in the genesis of GERD. Table I summarizes the mechanisms responsible for GERD in obese patients.

\section{Gastroesophageal reflux disease and laparoscopic sleeve gastrectomy}

Sleeve gastrectomy was first introduced in 1988 by Douglas Hess as the first stage of the biliopancreatic diversion with a duodenal switch procedure [18]. The encouraging weight loss outcomes combined with the laparoscopic approach contributed to the adaptation of LSG as a primary weight loss procedure [19]. In LSG, the stomach is divided vertically, along a line within the angle of His and a point along the greater curvature of the stomach between 
Table I. Mechanisms by which obesity leads to GERD

\begin{tabular}{|c|}
\hline Increased intraabdominal pressure \\
\hline Altered transdiaphragmatic pressure gradient \\
\hline Hiatal hernia \\
\hline Increased transient lower esophageal sphincter relaxations \\
\hline Improper esophageal clearance \\
\hline Dysmotility of the esophagus \\
\hline
\end{tabular}

3 and $6 \mathrm{~cm}$ from the pylorus, reducing the volume to approximately $25 \%$ of the original. This leaves the pyloric valve intact and preserves the continuity of the digestive tract [20]. In addition to its restrictive properties, LSG regulates the hunger sensation by the removal of entero-endocrine cells located in the fundus. This type of cell produces the appetite-stimulating hormone ghrelin. Ghrelin production is suppressed following LSG, leading to decreased stimulation of the hunger center [21].

There is still controversy in the literature regarding the relationship between GERD and LSG. Various mechanisms have been postulated to contribute to GERD (Table II).

The LSG modifies the anatomy of the gastroesophageal junction, changing it into a straight tubular segment and partially cutting the sling fibers that may affect the LES mechanism following dissection of the angle of His. The narrow stomach has been shown to have increased intragastric pressure on manometric measurements due to the excision of the fundus, which leads to a further increase in the gastroesophageal pressure gradient [22]. Braghetto et al. in a prospective study identified alterations in LES function after LSG. They performed esophageal manometry preoperatively and 6 months postoperatively in 20 patients. Postoperatively, the LES pressure decreased significantly and $85 \%$ of patients had hypotensive LES [23].

Herniation of the gastric sleeve into the thoracic cavity also encourages GERD. This can be caused by the disruption of the phrenoesophageal ligaments after dissection and also by the concomitant presence of an $\mathrm{HH}$ [24].

Redundant fundus left at the top of a sleeve can also dilate over time, resulting in GERD, and the shape of the sleeve itself can be a significant cause of GERD. In a study by Keidar et al., 8 patients had severe gas-
Table II. Mechanisms leading to GERD after LSG

\begin{tabular}{|c|}
\hline Modified anatomy of the gastroesophageal junction \\
\hline Disruption of the integrity of sling fibers \\
\hline Affected LES mechanism \\
\hline Narrowing stomach \\
\hline Increased intragastric pressure \\
\hline Dilation of the fundus \\
\hline Intrathoracic sleeve herniation \\
\hline Dissection of phrenoesophageal ligament \\
\hline Concomitant presence of a hiatal hernia
\end{tabular}

troesophageal dysmotility and reflux postoperatively. All of them had a dilated fundus with a narrowing of the mid-stomach [25]. In another study, Lazoura et al. included 85 consecutive morbidly obese patients who underwent LSG. Symptoms of GERD were evaluated preoperatively and at 1, 6, and 12 months postoperatively. The final shape of the sleeve was assessed by Gastrografin studies, performed in all patients on the third postoperative day. The tubular pattern was identified in $65.9 \%$ of patients, a superior pouch in $25.9 \%$ of patients, and an inferior pouch in $8.2 \%$ of patients. GERD symptoms intensity was higher in patients with the tubular pattern and a superior pouch compared to patients with an inferior pouch at 1,6 , and 12 months postoperatively. The authors concluded that an inferior pouch might be beneficial regarding GERD symptoms postoperatively [26]. In a retrospective chart review by Saleh et al., the lower pouch shape of the sleeve showed a trend toward less reflux and more weight loss [27]. It could be said that both weight loss and GERD outcomes associated with LSG have been found to vary by the final shape of the gastric sleeve.

Published data regarding the effects of LSG in patients with GERD are contradictory and comparison between studies is difficult.

Chiu et al. conducted a systematic review, analyzing the effects of LSG on GERD. There was discordance, with 4 studies showing an increase in GERD after LSG while 7 studies showed an improvement. They concluded that there was too much heterogeneity of the included studies and a final consensus could not be reached [28]. In another large systematic review, Stenard et al. included 25 studies, but the findings were again heterogeneous. Thirteen studies 
showed worsening of GERD after LSG, while 12 studies reported a favorable impact of LSG on GERD [29]. Oor et al. in a systematic review included 33 studies. Of these, 16 reported an increase and 12 a decrease in the prevalence of GERD symptoms after LSG. The authors concluded that there appeared to be a minimal trend towards an increased prevalence of GERD symptoms following LSG among the pooled studies, without any statistical difference [30].

Yet, there are some objective studies based on manometry and 24-hour pH monitoring or impedance that demonstrate an association between LSG and postoperative GERD. Del Genio et al. in a prospective study performed high-resolution manometry and 24-hour impedance-pH monitoring before and after LSG in 25 consecutive patients. At a median follow-up of 13 months, manometric findings showed unchanged LES function, increased ineffective peristalsis, and incomplete bolus transit. Impedance $\mathrm{pH}$ showed an increase in both acid exposure of the esophagus and the number of non-acid reflux events in postprandial periods [31]. In another prospective study by Burgerhart et al., 20 patients underwent esophageal function tests (high-resolution manometry and 24-hour impedance-pH monitoring) before and 3 months after LSG. Preoperative and postoperative symptoms were assessed using the Reflux Disease Questionnaire (RDQ). The results showed that GERD symptoms did not significantly change after LSG, but other upper gastrointestinal symptoms, particularly belching, epigastric pain, and vomiting, increased. The percentage of normal peristaltic contractions remained unchanged, but the LES pressure decreased. The authors concluded that after LSG, patients have significantly higher esophageal acid exposure [32]. Gemici et al. in a retrospective study performed 24-hour $\mathrm{pH}$ monitoring and esophageal manometry before and 3 months after LSG in 62 patients. The authors concluded that the LSG was found to cause a reduction in LES pressure, and an increase in acid reflux causing an extended relaxation time of the LES [33].

In the last years, the majority of published studies have suggested no improvement in symptoms or the development of new symptoms postoperatively (Table III) [34-40].

In a recent meta-analysis by Yeung et al. 46 studies totaling 10,718 patients who underwent LSG were included. Pooled data from 35 studies indicated a $19 \%$ increase in GERD symptoms between the preand postoperatively period. Also, for "de novo" GERD pooled data from 30 studies showed a $23 \%$ incidence in symptoms. Other postoperative outcomes included esophagitis at $30 \%, \mathrm{BE}$ at $6 \%$, rates of $\mathrm{HH}$ at $41 \%$, use of proton-pump inhibitors (PPIs) at 38\%, and conversion rate to Roux-en-Y gastric bypass (RYGB) of $4 \%$ due to severe reflux. The authors concluded that the postoperative prevalence of GERD, esophagitis, and BE following LSG was significant [41].

A multicenter study included 90 consecutive patients who underwent LSG and were evaluated through esophagogastroduodenoscopy (EGD). The prevalence of GERD symptoms significantly increased from $22 \%$ before surgery to $76 \%$ at the time of follow-up. EE and the usage of PPIs increased from $10 \%$ and $22 \%$ before LSG to $41 \%$ and $52 \%$, respectively, at the time of follow-up. Also, the prevalence of $\mathrm{BE}$ was $18.8 \%$, which represents a high rate [42].

Table III. Negative results of LSG on GERD in recent years

\begin{tabular}{|c|c|c|c|c|c|c|c|}
\hline Study & Type & Year & $\begin{array}{c}\text { No. of } \\
\text { patients }\end{array}$ & GERD evaluation & $\begin{array}{l}\text { Pre-LSG GERD } \\
\text { symptoms (\%) }\end{array}$ & $\begin{array}{l}\text { Post-LSG GERD } \\
\text { symptoms (\%) }\end{array}$ & $\begin{array}{l}\text { De novo } \\
\text { GERD (\%) }\end{array}$ \\
\hline Genco [34] & Prospective & 2017 & 110 & VAS, PPIs, EGD & 33.6 & 68.1 & - \\
\hline Mandeville [35] & Retrospective & 2017 & 100 & SR, PPIs, EGD & 17 & 50 & 47.8 \\
\hline Georgia [36] & Prospective & 2017 & 12 & 24-h pH-m & - & 83.3 & 50 \\
\hline $\operatorname{Lim}[37]$ & Retrospective & 2019 & 63 & RDQ, EGD & 31.7 & 47.6 & 31.7 \\
\hline Viscido [38] & Prospective & 2018 & 109 & RDQ, EGD & 33 & 44 & 36.9 \\
\hline Soricelli [39] & Prospective & 2018 & 144 & EGD, VAS, PPIS & 40.9 & 70.2 & 36.8 \\
\hline Borbely [40] & Prospective & 2019 & 222 & $\begin{array}{l}\text { RDQ, EGD, E, } \\
24-h \mathrm{pH}-\mathrm{m}\end{array}$ & 23 & 52 & 73 \\
\hline
\end{tabular}

VAS - visual analogue scale, PPIs - proton-pump inhibitors, EGD - esophagogastroduodenoscopy, SR - symptoms reported by patients, 24-h pH-m - 24 hour pH monitoring, $R D Q$ - reflux disease questionnaire, $E$ - esophagograms. 
Regarding BE, Genco et al. included 110 patients who were submitted to LSG and found alarming evidence. They performed EGD preoperatively and at a 5-year follow-up. In the EGD findings 19 (17.2\%) patients had newly diagnosed BE. They concluded that postoperative surveillance should be mandatory regardless of the presence or absence of GERD symptoms because the incidence of BE was not related to GERD symptoms. Also, the incidence of GERD symptoms and PPI intake significantly increased from $33.6 \%$ to $68.1 \%$ and $19.1 \%$ to $57.2 \%$ respectively in the postoperative period [34].

Regarding the investigation of GERD, there is no consensus. Studies used a variety of methods to investigate and define GERD. This varies from clinical notes to validated questionnaires, to objective investigations, EGD, or a combination of the above [41].

While the majority of the studies point toward a higher prevalence of GERD after LSG, a few studies still show some improvement or at least maintenance of the same level of preexisting GERD (Table IV) [43-45]. Theoretically, LSG can promote the improvement of GERD by reversal of the weight and visceral adiposity and lead to a decrease in intra-abdominal pressure. Also reduced acid production secondary to resection of the fundus, accelerated gastric emptying, and reduced gastric volume plays a role in the improvement of GERD outcomes [28, 29]. Daes et al. observed a significant decrease in GERD symptoms by avoiding a narrowing at the junction of the vertical and the horizontal portions of the sleeve, removing almost all of the fundus and routinely repairing the $\mathrm{HH}$. The authors concluded that careful attention to the operative technique can reduce the appearance of GERD symptoms 6-12 months after LSG [43].

\section{Laparoscopic sleeve gastrectomy and concomitant hiatal hernia repair}

Another controversial aspect regarding GERD in obese patients involves the identification and simul- taneous repair of $\mathrm{HH}(\mathrm{HHR})$ during LSG. The prevalence of $\mathrm{HH}$ in the obese population is increased, with a range between 40 and 50\% [17]. In a systematic review of research on concomitant HHR and LSG, 16 out of 17 studies recommended simultaneous repair of HH during LSG [46]. Soricelli et al. included in their study 378 obese patients who underwent LSG. Ninety-seven (25.6\%) out of 378 patients underwent LSG and concomitant HHR. Preoperatively $\mathrm{HH}$ was diagnosed in 42 (11.1\%) patients and symptomatic GERD was present in 60 (15.8\%) patients. In 55 (14.5\%) patients $\mathrm{HH}$ was diagnosed intraoperatively. No cases of "de novo" GERD symptoms were seen when LSG was associated with concomitant HHR [47]. In another study, Sheppard et al. reported that there were no significant differences in reflux rates between patients with and without an HHR during LSG [48].

However, less satisfactory outcomes have been reported also. In a study by Santonicola et al., the authors found an increase in GERD symptoms after concomitant LSG and HHR. Of 78 patients with LSG and HHR, 38.4\% reported GERD symptoms and $43.3 \%$ reported postoperative persistence of GERD symptoms. Also, 22.9\% developed "de novo" GERD after LSG and HHR. The authors concluded that concomitant HHR did not lead to an improvement in GERD symptoms and even resulted in a higher heartburn intensity-frequency score [49].

Further prospective studies are necessary to provide adequate information regarding the additional effect of concomitant HHR on GERD symptoms, in patients submitted to LSG.

\section{Laparoscopic sleeve gastrectomy compared with Roux-en-Y gastric bypass}

At first sight, patients having LSG appear to have a higher risk of GERD than patients having RYGB. Therefore, it is important to emphasize the difference between the two most widely used bariatric

Table IV. Studies showing improvement of GERD after LSG

\begin{tabular}{|lcccccc|}
\hline Study & Type & Year & $\begin{array}{c}\text { No. of } \\
\text { patients }\end{array}$ & GERD evaluation & $\begin{array}{c}\text { Pre-LSG GERD } \\
\text { symptoms (\%) }\end{array}$ & $\begin{array}{c}\text { Post-LSG GERD } \\
\text { symptoms (\%) }\end{array}$ \\
\hline Daes [43] & Prospective & 2012 & 134 & SR, EGD & 49.2 & 1.5 \\
\hline Daes [44] & Prospective & 2014 & 382 & SR, EGD & 44.5 & 2.6 \\
\hline Sucandy [45] & Retrospective & 2015 & 131 & SR, EGD & 51 & 22.2 \\
\hline
\end{tabular}

SR - symptoms reported by patients, EGD - esophagogastroduodenoscopy. 
surgical methods (LSG versus RYGB) regarding GERD and the risk of GERD after surgery. To date, however, there is no consensus in this regard.

In a recent meta-analysis, Gu et al. investigated the relationship between LSG compared with RYGB and GERD. Twenty-three studies were included, and the results showed that LSG was associated with a higher risk of GERD than RYGB. Compared with LSG, obese patients who received RYGB had a lower risk of new-onset or worsened GERD. The authors recommended RYGB as the preferred treatment for obese patients with GERD [50].

In the Swiss Multicenter Bypass or Sleeve Study (SM-BOSS), which is a randomized controlled trial comparing RYGB and LSG, 217 patients were enrolled (110 in the RYGB group and 107 in the LSG group). GERD remission was observed in $60.4 \%$ of patients after RYGB and in only $25 \%$ of patients after LSG, whereas GERD worsened in $31.8 \%$ of patients after LSG compared with $6.3 \%$ of patients after RYGB. At 5 years, "de novo" GERD appeared in $31.6 \%$ of LSG patients versus $10.7 \%$ of RYGB patients. The conclusion was that GERD symptoms were significantly more frequent after LSG compared to RYGB [51].

In another recent nonrandomized clinical trial comparing the incidence of GERD after LSG and RYGB, 30 patients were included in the LSG group, and 16 patients in the RYGB group. The DeMeester score increased from 10.9 to 40.2 in the LSG group compared with the RYGB group, where the DeMeester score increased from 9.5 to 12.2. The authors concluded that the high incidence of GERD after LSG represent a contraindication for this type of procedure, while RYGB remains the preferred method for patients with GERD [52].

Biter et al. in a randomized controlled trial comparing QoL (quality of life) after LSG versus RYGB, with a focus on GERD, found that the mean GerdQ (Gastroesophageal Reflux Disease Questionnaire) score was 6.95 in the LSG group versus 5.5 in the RYGB group after 2 months of follow-up. After 12 months, the mean GerdQ score was 6.63 in the LSG group versus 5.6 in the RYGB group. The trial concluded that patients in the LSG group had significantly higher GerdQ scores at both 2 and 12 months postoperatively than patients in the RYGB group [53].

The majority of studies regarding this subject suggest that LSG should not be the procedure of choice in patients with GERD symptoms.

\section{Conclusions}

This review discusses the topics surrounding the relationship between GERD and LSG.

In recent literature, LSG is associated with an increased incidence of GERD, which is concerning, considering its increased popularity globally. A disadvantage in the diagnosis of GERD is the heterogeneity in defining it. Some of the studies rely only on symptoms reported by patients, so studies that use this indicator are prone to subject bias. Studies that rely on objective measurements such as functional tests and endoscopic evaluation probably represent the best method to make an official diagnosis of GERD.

Further randomized controlled trials using both standardized questionnaires and objective esophageal functional tests are needed and provision of long-term follow-up is necessary to provide adequate information regarding the relationship between LSG and GERD. Also, the final shape of the LSG appears to play an important role in the GERD outcomes, postoperatively.

In conclusion, bariatric and general surgeons performing LSG must be aware of the existence of GERD. Also, patients should be informed regarding the possible effects of LSG on GERD, including the development of "de novo" GERD symptoms in the postoperative period.

\section{Conflict of interest}

The authors declare no conflict of interest.

\section{References}

1. Vakil N, van Zanten SV, Kahrilas P, et al. The Montreal definition and classification of gastroesophageal reflux disease: a global evidence - based consensus. Am J Gastroenterol 2006; 101 : 1900-20.

2. El-Serag HB, Sweet S, Winchester CC, et al. Update on the epidemiology of gastro-oesophageal reflux disease: a systematic review. Gut 2014; 63: 871-80.

3. Mikami DJ, Murayama KM. Physiology and pathogenesis of gastroesophageal reflux disease. Surg Clin N Am 2015; 95 : 515-25.

4. Hampel H, Abraham NS, El-Serag HB. Meta-analysis: obesity and the risk for gastroesophageal reflux disease and its complications. Ann Intern Med 2005; 143: 199-211.

5. Gonzalez-Muniesa P, Martinez-Gonzalez MA, Hu FB, et al. Obesity. Nat Rev Dis Primers 2017; 3: 17034.

6. Heymsfield SB, Wadden TA. Mechanism, pathophysiology and management of obesity. N Engl J Med 2017; 376: 254-66.

7. El-Hadi M, Birch DW, Gill RS, et al. The effect of bariatric surgery on gastroesophageal reflux disease. Can J Surg 2014; 57 : 139-44. 
8. Gagner M, Hutchinson C, Rosenthal R. Fifth International Consensus Conference: current status of sleeve gastrectomy. Surg Obes Relat Dis 2016; 12: 750-6.

9. Ali M, El Chaar M, Ghiassi S, et al. American Society for Met abolic and Bariatric Surgery updated position statement on sleeve gastrectomy as a bariatric procedure. Surg Obes Relat Dis 2017; 13: 1652-7.

10. Melissas J, Braghetto I, Molina JC, et al. Gastroesophageal reflux disease and sleeve gastrectomy. Obes Surg 2015; 25: 2430-5.

11. Horsley T. Tips for improving the writing and reporting quality of systematic, scoping and narrative reviews. J Contin Educ Health Prof 2019; 39: 54-7.

12. Chang P, Friedenberg F. Obesity and GERD. Gastroenterol Clin North Am 2014; 43: 161-73.

13. Braghetto I, Korn O, Csendes A, et al. Laparoscopic treatment of obese patients with gastroesophageal reflux disease and Barrett's esophagus: a prospective study. Obes Surg 2012; 22: 764-72.

14. Nadaleto BF, Herbella FAM, Patti MG. Gastroesophageal reflux disease in the obese: pathophysiology and treatment. Surgery 2016; 159: 475-86.

15. Ayazi S, Hagen JA, Chan LS, et al. Obesity and gastroesophageal reflux: quantifying the association between body mass index, esophageal acid exposure, and lower esophageal sphincter status in a large series of patients with reflux symptoms. J Gastrointest Surg 2009; 13: 1440-7.

16. Kuper MA, Kramer KM, Kirschniak A, et al. Dysfunction of the lower esophageal sphincter and dysmotility of the tubular esophagus in morbidly obese patients. Obes Surg 2009; 19: 1143-9.

17. Che F, Nguyen B, Cohen A, et al. Prevalence of hiatal hernia in the morbidly obese. Surg Obes Relat Dis 2013; 9: 920-4.

18. Hess DS, Hess DW. Biliopancreatic diversion with a duodenal switch. Obes Surg 1998; 8: 267-82.

19. Neagoe R, Muresan M, Timofte D, et al. Long-term outcomes of laparoscopic sleeve gastrectomy - a single-center prospective observational study. Videosurgery Miniinv 2019; 14: 242-8.

20. Akkary E, Duffy A, Bell R. Deciphering the sleeve: technique, in dications, efficacy, and safety of sleeve gastrectomy. Obes Surg 2008; 18: 1323-9.

21. Sharma G, Nain PS, Sethi P, et al. Plasma ghrelin levels after laparoscopic sleeve gastrectomy in obese individuals. Indian J Med Res 2019; 149: 544-7.

22. Mion F, Tolone S, Garros A, et al. High-resolution impedance manometry after sleeve gastrectomy: increased intragastric pressure and reflux are frequent events. Obes Surg 2016; 26: 2449-56.

23. Braghetto I, Lanzarini E, Korn O, et al. Manometric changes of the lower esophageal sphincter after sleeve gastrectomy in obese patients. Obes Surg 2010; 20: 357-62.

24. Hutopila I, Copaescu C. Hiatal hernia is more frequent than expected in bariatric patients. Intraoperative findings during laparoscopic sleeve gastrectomy. Chirurgia 2019; 114: 779-89.

25. Keidar A, Appelbaum L, Schweiger C, et al. Dilated upper sleeve can be associated with severe postoperative gastroesophageal dysmotility and reflux. Obes Surg 2010; 20: 140-7.

26. Lazoura O, Zacharoulis D, Triantafyllidis G, et al. Symptoms of gastroesophageal reflux following laparoscopic sleeve gastrec- tomy are related to the final shape of the sleeve as depicted by radiology. Obes Surg 2011; 21: 295-9.

27. Saleh AA, Janik MR, Mustafa RR, et al. Does sleeve shape make a difference in outcomes? Obes Surg 2018; 28: 1731-7.

28. Chiu S, Birch DW, Shi X, et al. Effect of sleeve gastrectomy on gastroesophageal reflux disease: a systematic review. Surg Obes Relat Dis 2011; 7: 510-5.

29. Stenard F, lannelli A. Laparoscopic sleeve gastrectomy and gastroesophageal reflux. World J Gastroenterol 2015; 21: 10348-57.

30. Oor JE, Roks DJ, Cagdus U, et al. Laparoscpic sleeve gastrectomy and gastroesophageal reflux disease: a systematic review and meta-analysis. Am J Surg 2016; 211: 250-67.

31. Del Genio G, Tolone S, Limongelli P, et al. Sleeve gastrectomy and development of "de novo" gastroesophageal reflux. Obes Surg 2014; 24: 71-7.

32. Burgerhart JS, Schotborgh CAI, Schoon EJ, et al. Effect of sleeve gastrectomy on gastroesophageal reflux. Obes Surg 2014; 24: 1436-41.

33. Gemici E, Kones O, Seyit H. Outcomes of laparoscopic sleeve gastrectomy by means of esophageal manometry and $\mathrm{pH}$-metry, before and after surgery. Videosurgery Mininv 2020; 15 : 129-35.

34. Genco A, Soricelli E, Casella G, et al. Gastroesophageal reflux disease and Barrett's esophagus after laparoscopic sleeve gastrectomy: a possible, underestimated long-term complications. Surg Obes Relat Dis 2017; 13: 568-74.

35. Mandeville Y, Van Looveren R, Vancoillie PJ, et al. Moderating the enthusiasm of sleve gastrectomy: up to fifty percent of reflux symptoms after ten years in a consecutive series of one hundred laparoscopic sleeve gastrectomies. Obes Surg 2017; 27: 1797-803.

36. Georgia D, Stamatina T, Maria N, et al. 24-h Multichannel intraluminal impedance $\mathrm{PH}$-metry 1 year after laparocopic sleeve gastrectomy: an objective assessment of gastroesophageal reflux disease. Obes Surg 2017; 27: 749-53.

37. Lim CH, Lee PC, Lim E, et al. Correlation between symptomatic gastro-esophageal reflux disease (GERD) and erosive esophagitis (EE) post-vertical sleeve gastrectomy (VSG). Obes Surg 2019; 29: 207-14.

38. Viscido G, Gorodner V, Signorini F, et al. Laparoscopic sleeve gastrectomy: endoscopic findings and gastroesophageal reflux symptoms at 18-months follow-up. J Laparoendosc Adv Surg Tech A 2018; 28: 71-7.

39. Soricelli E, Casella G, Baglio G, et al. Lack of correlation between gastroesophageal reflux disease symptoms and esophageal lesions after sleeve gastrectomy. Surg Obes Relat Dis 2018; 14: 751-6.

40. Borbely Y, Schaffner E, Zimmermann L, et al. De novo gastroesophageal reflux disease after sleeve gastrectomy: role of preoperative silent reflux. Surg Endosc 2019; 33: 789-93.

41. Yeung KTD, Penney N, Ashrafian L, et al. Does sleeve gastrectomy expose the distal esophagus to severe reflux? A systematic review and meta-analysis. Ann Surg 2020; 271: 257-65.

42. Sebastianelli L, Benois M, Vanbiervliet G, et al. Systematic endoscopy 5 years after sleeve gastrectomy results in a high rate of Barrett's esophagus: results of a multicenter study. Obes Surg 2019; 29: 1462-9. 
43. Daes J, Jimenez ME, Said N, et al. Laparoscopic sleeve gastrectomy: symptoms of gastroesophageal reflux can be reduced by changes in surgical technique. Obes Surg 2012; 22: 1874-9.

44. Daes J, Jimenez ME, Said N, et al. Improvement of gastroesophageal reflux symptoms after standardized laparoscopic sleeve gastrectomy. Obes Surg 2014; 24: 536-40.

45. Sucandy I, Chrestiana D, Bonanni F, et al. Gastroesophageal reflux symptoms after laparoscopic sleeve gastrectomy for morbid obesity. The importance of preoperative evaluation and selection. North Am J Med Sci 2015; 7: 189-93.

46. Mahawar KK, Carr WRJ, Jennings N, et al. Simultaneous sleeve gastrectomy and hiatus hernia repair: a systematic review. Obes Surg 2015; 25: 159-66.

47. Soricelli E, lossa A, Casella G, et al. Sleeve gastrectomy and crural repair in obese patients with gastroesophageal reflux disease and/or hiatal hernia. Surg Obes Relat Dis 2013; 9: 356-61.

48. Sheppard CE, Sadowski DC, de Gara CJ, et al. Rates of reflux before and after laparoscopic sleeve gastrectomy for severe obesity. Obes Surg 2015; 25: 763-8.

49. Santonicola A, Angrisani L, Cutolo P, et al. The effect of laparoscopic sleeve gastrectomy with or without hiatal hernia repair on gastroesophageal reflux disease in obese patients. Surg Obes Relat Dis 2014; 10: 250-5.

50. Gu L, Chen B, Du N, et al. Relationship between bariatric surgery and gastroesophageal reflux disease: a systematic review and meta-analysis. Obes Surg 2019; 29: 4105-13.

51. Peterli R, Wolnerhanssen BK, Peters T, et al. Effect of laparoscopic sleeve gastrectomy vs laparoscopic Roux-en-Y gastric bypass on weight loss in patients with morbid obesity: the SM-BOSS randomized clinical trial. JAMA 2018; 319: 255-65.

52. Raj PP, Bhattacharya S, Misra S, et al. Gastroesophageal reflux-related physiologic changes after sleeve gastrectomy and Roux-en-Y gastric bypass: a prospective comparative study. Surg Obes Relat Dis 2019; 15: 1261-9.

53. Biter LU, van Buuren MMA, Mannaerts GHH, et al. Quality of Life 1 year after laparoscopic sleeve gastrectomy versus laparoscopic Roux-en-Y gastric bypass: a randomized controlled trial focusing on gastroesophageal reflux disease. Obes Surg 2017; 27: 2557-65.

Received: 3.01.2021, accepted: 18.01.2021. 